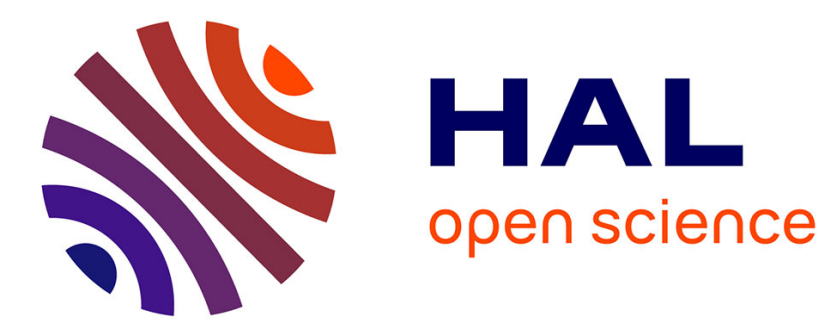

\title{
Deblurring of irregularly sampled images by TV regularization in a spline space
}

\author{
Andrés Almansa, Julien Caron, Sylvain Durand
}

\section{To cite this version:}

Andrés Almansa, Julien Caron, Sylvain Durand. Deblurring of irregularly sampled images by TV regularization in a spline space. (ICIP) International Conference on Image Processing, Sep 2010, Hong Kong, China. pp.1181-1184. hal-00497000

\section{HAL Id: hal-00497000 https://hal.science/hal-00497000}

Submitted on 2 Jul 2010

HAL is a multi-disciplinary open access archive for the deposit and dissemination of scientific research documents, whether they are published or not. The documents may come from teaching and research institutions in France or abroad, or from public or private research centers.
L'archive ouverte pluridisciplinaire HAL, est destinée au dépôt et à la diffusion de documents scientifiques de niveau recherche, publiés ou non, émanant des établissements d'enseignement et de recherche français ou étrangers, des laboratoires publics ou privés. 


\title{
DEBLURRING OF IRREGULARLY SAMPLED IMAGES BY TV REGULARIZATION IN A SPLINE SPACE
}

\author{
A. Almansa ${ }^{\dagger}$, J. Caron*, S. Durand \\ Telecom ParisTech \\ - University of Picardie Jules Verne - \\ LAMFA CNRS UMR 6140 \\ University Paris Descartes \\ MAP5 CNRS UMR 8145
}

Copyright 2010 IEEE. Published in the IEEE 2100 International Conference on Image Processing (ICIP 2010), scheduled for September 26-29, 2010 in Hong Kong. Personal use of this material is permitted. However, permission to reprint/republish this material for advertising or promotional purposes or for creating new collective works for resale or redistribution to servers or lists, or to reuse any copyrighted component of this work in other works, must be obtained from the IEEE. Contact: Manager, Copyrights and Permissions / IEEE Service Center / 445 Hoes Lane / P.O. Box 1331 / Piscataway, NJ 08855-1331, USA. Telephone: + Intl. 908-562-3966.

\begin{abstract}
Restoring a regular image from irregular samples was shown feasible via quadratic regularization using Fourier [1] and spline [2] representations. When the image is also blurred and noisy (as is usually the case in satellite imaging) $\ell^{1}$ regularizers (like TV) were shown most effective [3], but their Fourier-domain implementation has a prohibitive computational cost. We present here a new method that combines a spline representation (for speed) with TV regularization to obtain a more accurate and good-quality restored image. Extending this approach to the blurred case is not as trivial as in the Fourier representation. Indeed, in order to avoid the sampling operator to lose its sparse structure, a projection of the convolution operator on a spline space becomes necessary. Extensive experimental results with automatic regularization and stopping criteria show that our method achieves the accuracy of [3] with much less computational cost, closer to [2].
\end{abstract}

Index Terms - Image Restoration, Image Sampling, Spline functions, Satellite Applications, Variational Methods

\section{INTRODUCTION}

The pin-hole camera model with a regular sampling grid on the image plane is widely accepted and used in computer vision. We keep that model here as the ideal representation of an image, that we want to restore from a more complex acquisition system. In fact, in certain cases (wide-angle lenses, vibrating push-broom earth observation systems, medical devices) either the scene or the sampling grid are distorted. In either case we can model the acquisition process as a pin-hole camera with a blurring kernel and a perturbed sampling grid (a particular kind of irregular sampling). The observation grid is the set $\Lambda$ of points $M_{i, j}\left(x_{i, j}, y_{i, j}\right)$, such that $x_{i, j}=i+\epsilon_{x}(i, j)$ and $y_{i, j}=j+\epsilon_{y}(i, j) . \epsilon_{x}$ and $\epsilon_{y}$ are smooth, small perturbation functions that we assume to be known. Data are denoted by $Z_{i, j}$ and correspond to the values of a function $u$, convolved with a point spread function $h$, at location $M_{i, j}$. As data may be noisy, we consider i.i.d. Gaussian variables $\mathcal{N}_{i, j}$ with zero mean and variance $\sigma^{2}$ (see fig. 1 (b) p. 3)

$$
Z_{i, j}=(h * u)\left(x_{i, j}, y_{i, j}\right)+\mathcal{N}_{i, j}
$$

Finding $u$ from a set $\Lambda$ and a vector $Z$ is an ill-posed problem because of (i) the non-uniqueness of the solution and (ii) instability phenomena. First works on this topic, in dimension one, assume that $u$ is a trigonometric polynomial. In this case the sampling equation is the linear system $Z=S_{\Lambda} \cdot \hat{H} \cdot \hat{U}+\mathcal{N}$, where $U$ is the regular image $\left(U_{i, j}=u(i, j)\right), \hat{H}$ is the diagonal matrix associated to $\hat{h}$ and $S_{\Lambda}$ is the sampling matrix acting on Fourier coefficients : $\left(S_{\Lambda} \cdot \hat{U}\right)_{i, j}=u\left(x_{i, j}, y_{i, j}\right)$. The sampling matrix is such that $S_{\Lambda}^{*} \cdot S_{\Lambda}$ is a Toeplitz matrix, and one of the first methods in irregular sampling (ACT [1]) takes advantage of this property. It solves the normal equations associated to the least-squares minimization problem by a conjugate gradient,

\footnotetext{
${ }^{\dagger}$ Thanks to CNES (French space agency), FREEDOM (ANR07-JCJC-0048-01), Callisto (ANR-09-CORD-003), and ECOS Sud U06E01 projects for funding

*Thanks to CNRS agency and Picardie Region for funding.
} 
computing the matrix-vector multiplications in $O\left(N \log _{2}(N)\right)$ operations via the Fast Fourier Transform ( $N$ is the size of the signal). Even when invertible, this linear system can be ill-conditioned, the conjugate gradient then has a poor convergence rate and produces a signal corrupted with artifacts. That is why Feichtinger et al. proposed to improve conditioning with a diagonal local weights matrix $W_{1}$ and regularized the least-squares problem with the square-norm of a weighted Fourier coefficients vector $W_{2} \cdot C(\mathrm{ACT}+\mathrm{R})$

$$
\min _{C} \quad\left\|W_{2} \cdot C\right\|^{2}+\lambda .\left\|W_{1} \cdot\left(S_{\Lambda} \cdot H \cdot C-Z\right)\right\|^{2}
$$

In recent works about perturbed sampling [4][3], the authors exploit the idea of Fourier weights and the good results of the total variation regularizer in image deblurring. They replaced the first term in (2) by the total variation (ACT+TV [4]), and also experimented the norm of a derivative-like vector with adapted Fourier coefficients (ACT+FAR [3]) and a local-regularization method, based on a local noise estimator. These methods are very efficient, but the successive improvements (non quadratic regularizers and local constraints) have increased the computational complexity of restoration and faster methods have to be developed to process large images. As proposed by Unser et al. in [2], we will assume that the function $u$ is a spline, which results in a sparse sampling operator $S_{\Lambda}$ and a $O(N)$ computation cost for the matrix-vector multiplications. Our variational formulation, based on discrete total variation, is solved by a Forward-Backward algorithm [5] and uses the TV proximal operator (Chambolle's algorithm [6]).

\section{VARIATIONAL FORMULATION}

\subsection{Spline approximation}

Regular B-splines of order $D \geq 0$, denoted by $\beta^{(D)}$, are piecewise polynomial functions on $\mathbf{R}$ with $D-1$ smoothness degree and support in $\left[-\frac{D+1}{2}, \frac{D+1}{2}\right]$. They are defined by $\beta^{(0)}=\mathbb{1}_{\left[-\frac{1}{2}, \frac{1}{2}\right]}$ and

$$
\beta^{(D+1)}=\beta^{(D)} * \mathbb{1}_{\left[-\frac{1}{2}, \frac{1}{2}\right]}
$$

with $\mathbb{1}_{\left[-\frac{1}{2}, \frac{1}{2}\right]}(\cdot)$ the indicator function of segment $\left[-\frac{1}{2}, \frac{1}{2}\right]$. We work with the tensorial product of $\beta^{(3)}(x)$ and $\beta^{(3)}(y)$ (cubic splines), which we simply denote by $\beta^{(3)}(x, y)$, and the approximation space $V$ is the vectorial space spanned by translations of $\beta^{3}(x, y)$ by a unit step in each direction. We periodize it with periods $(n, 0)$ and $(0, m)$ (original image dimensions) and further consider periodic convolution for $h$.

Irregular samples of a function in $V$ are linear combinations of its coefficients $C_{i, j}$ in the bicubic splines basis, and the sampling operator on $\mathbf{R}^{n} \times \mathbf{R}^{m}$ has the sparse form

$$
S_{\Lambda}((i, j),(k, l))=\beta^{(3)}\left(x_{i, j}-k, y_{i, j}-l\right)
$$

\subsection{Adapted constrained formulation}

This operator may also be non-invertible or ill-conditioned, so we adopt a constrained formulation and minimize the discrete total variation of $u$, such that the differences between $(h * u)$ and $Z$, at locations $M_{i, j}$, have a square norm no larger than the noise variance $\sigma^{2}$ times the number of samples $m . n$

$$
\begin{array}{ll} 
& \min _{u \in V} T V(u) \\
\text { s.t. } & \sum_{i, j}\left|(h * u)\left(x_{i, j}, y_{i, j}\right)-Z_{i, j}\right|^{2} \leq m . n . \sigma^{2}
\end{array}
$$

At this point the formulation does not meet our need for a sparse and easily computable matrix $S_{\Lambda}$ because $(h * u)$ might not be a spline or have a convenient analytical expression. This is why we replace it with its projection on $V$. By linearity, shift invariance and periodicity, we only have to compute one projection

$$
\begin{aligned}
\operatorname{proj}_{V}\left(h * \beta^{(3)}\right) & =\operatorname{argmin}_{v \in V}\left\|h * \beta^{(3)}-v\right\|_{L^{2}(V)}^{2} \\
& =\sum_{i, j} \tilde{h}[i, j] \beta_{i, j}^{(3)}
\end{aligned}
$$


with $\beta_{i, j}^{(3)}(x, y)=\beta^{(3)}(x-i, y-j)$. Coefficients $\tilde{h}[i, j]$ solve the following $m . n \times m . n$ linear system

$$
\sum_{i, j} \tilde{h}[i, j]\left\langle\beta_{i, j}^{(3)}, \beta_{k, l}^{(3)}\right\rangle=\left\langle h * \beta^{(3)}, \beta_{k, l}^{(3)}\right\rangle \quad \forall k, l
$$

Since $\left\langle\beta_{i, j}^{(3)}, \beta_{k, l}^{(3)}\right\rangle=\left\langle\beta^{(3)}, \beta_{k-i, l-j}^{(3)}\right\rangle$, it can be solved with Fourier transforms if both scalar products are known. The first term is a classic computation with splines and is equal to $\beta^{(7)}(k-i, l-j)$ (with care to the periodicity). The second term must be computed in the Fourier domain with Plancherel's formula, i.e. by an infinite sum of Fourier coefficients

$$
\begin{aligned}
& \left\langle h * \beta^{(3)}, \beta_{k, l}^{(3)}\right\rangle_{L^{2}(V)}=\left\langle\hat{h} \widehat{\beta^{(3)}}, \widehat{\beta_{k, l}^{(3)}}\right\rangle_{l^{2}(Z)} \\
= & \sum_{q, r \in \mathbf{Z}} \hat{h}\left|\widehat{\beta^{(3)}}\right|^{2}\left(\frac{2 \pi q}{n}, \frac{2 \pi r}{m}\right) \cdot e^{i\left(\frac{2 \pi q \cdot k}{n}+\frac{2 \pi r . l}{m}\right)}
\end{aligned}
$$

The fast decay of $\hat{h} \cdot\left|\widehat{\beta^{(3)}}\right|^{2}$ allows the computation of a very good approximation for indices varying in $[-2 n+1,2 n] \times$ $[-2 m+1,2 m]$ but this can be done with any accuracy between $[-K n+1, K n] \times[-K m+1, K m]$ for $K$ large enough. This approximation is the decimated discrete Fourier transform of a $2 K n \times 2 K m$ signal, and finally the coefficients $\tilde{h}[i, j]$ are obtained by division in the discrete Fourier domain.

The constrained problem, with the same weights $W_{1}$ as in (2), takes the following Lagrangian form

$$
\min _{C} T V\left(S_{\mathbf{Z}^{2}} \cdot C\right)+\lambda \cdot\left\|W_{1} \cdot\left(S_{\Lambda} \cdot \tilde{H} \cdot C-Z\right)\right\|^{2}
$$

By the change of variable $U=S_{\mathbf{Z}^{2}}$.C (the regularly sampled image) it is equivalent to the following problem

$$
\min _{U} \underbrace{T V(U)+\lambda \cdot\left\|W_{1} \cdot\left(S_{\Lambda} \cdot \tilde{H} \cdot\left(S_{\mathbf{Z}^{2}}\right)^{-1} \cdot U-Z\right)\right\|^{2}}_{E_{1}(\lambda, U)}
$$

This problem always has a solution, but the minimizer is generally not unique due to the non strict convexity of the total variation. Nevertheless, all solutions have the same level lines and the data fitting term generally imposes uniqueness in most parts of the image.

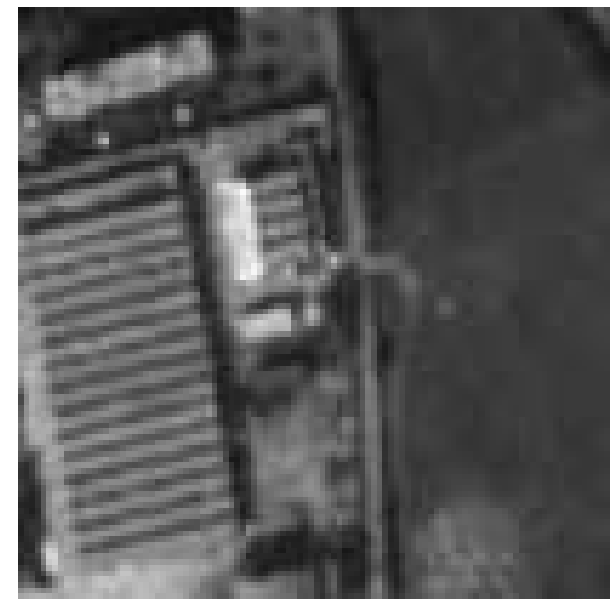

(a) Reference

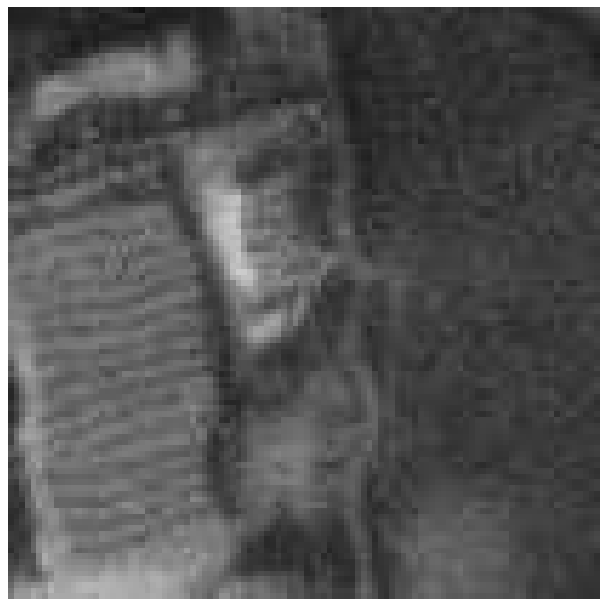

(b) Data

Fig. 1. Detail of reference and data with $\sigma=5$. 


\section{FROM VARIATIONAL FORMULATION TO FORWARD-BACKWARD ALGORITHM}

Our problem belongs to the more general class of problems treated by Combettes et al. in [5], namely

$$
\min _{U} \quad f_{1}(U)+f_{2}(U)
$$

with $f_{1}$ the discrete total variation and $f_{2}$ the data fitting term. They are both proper, lower semi-continuous convex functions and $f_{2}$ is differentiable with affine gradient. We refer the reader to [5] for convex theory and particularly proximal operators, which were first introduced by Moreau in the 60s [7].

The proximal operator we use here is the $T V-l_{2}$ denoiser with parameter $\gamma>0$

$$
\operatorname{prox}_{(T V, \gamma)}(U)=\operatorname{argmin}_{V} \underbrace{T V(V)+\frac{1}{2 \gamma} \cdot\|V-U\|^{2}}_{E_{2}(U, V)}
$$

It has been extensively studied and many algorithms have been developed to solve this minimization problem. One of the fastest and simplest is Chambolle's well known algorithm [6] which uses a dual formulation and solves a projection problem in the dual variable. There exist faster algorithms in the case of anisotropic total variation using graph cuts, which would yield similar results, but in our case $\gamma$ stays small and Chambolle's algorithm is very fast. Problem (5) is equivalent to the following equation (with $L=S_{\Lambda} \cdot \tilde{H} \cdot S_{\mathbf{Z}^{2}}^{-1}$ ) (see [5])

$$
U=\operatorname{prox}_{(T V, \gamma)}\left(U-2 \cdot \gamma \cdot \lambda \cdot L^{*} \cdot W_{1}^{2} \cdot(L \cdot U-Z)\right)
$$

The Forward-Backward algorithm associated to problem (5) is the fixed point iteration of eq. (8). It only involves the proximal operator (7) of the total variation and the gradient of the data fitting term

$$
\left\{\begin{array}{l}
U^{(k+1 / 2)}=U^{(k)}-2 \cdot \gamma \cdot \lambda \cdot L^{*} \cdot W_{1}^{2} \cdot\left(L \cdot U^{(k)}-Z\right) \\
U^{(k+1)}=\operatorname{prox}_{(T V, \gamma)}\left(U^{(k+1 / 2)}\right)
\end{array}\right.
$$

Articles [5] and [8] provide theoretical results of convergence for this algorithm, which can be applied in our case. Its convergence speed has been studied in the case of a variable step $\gamma$ (Nesterov [9]) and is then a $O\left(\frac{1}{k}\right)$.

\subsection{Experiments}

\subsubsection{Implementation}

We implemented this algorithm in C and used the FFTW library for fast convolutions. A first initial guess of $\lambda$ is obtained by a simple truncated conjugate gradient (see section 3.1.3), which gives an approximate value of $\lambda$ and $U$, and accelerates our Uzawa loop. Parameter $\gamma$ is estimated by an iterative scheme, ensuring stability and convergence (it depends on $\lambda$ and the largest eigenvalue of $\left.L^{*} . W_{1}^{2} \cdot L\right)$.

The algorithm contains three nested loops (i) Chambolle's algorithm, (ii) fixed point and (iii) Uzawa's loop on $\lambda$. The first two loops automatically stop with decreasing energy. A good stopping criteron for theses variational problems is a relative decay rate of $1.10^{-5}$ for $E_{2}\left(U, V^{k}\right)$ (see eq. (7)) and $1.10^{-4}$ for $E_{1}\left(\lambda, U^{k}\right)$ (see eq. (5)).

Uzawa's loop tunes $\lambda$ such that equality holds in (4), because the solution lies on the boundary of the constraint (except in the very special case of nearly constant images). The parameter $\lambda$ is updated with a multiplicative method [10] until (4) is verified up to a small tolerance. 


\subsubsection{Simulations}

We simulated realistic irregular acquisition of a continuous scene, by applying a low-pass filter to a high resolution image and then sampling on $\Lambda$. This low-pass filtering allows for irregular sampling and sub-sampling with a small alias.

In the simple unblurry case we try to restore irregular samples from a twice higher resolution image convolved with a 0.5 cut-off prolate. The continuous underlying model we adopted is trigonometric polynomials. The reference image (see fig. 1 (a) ) is obtained by convolving the high resolution image with the prolate and subsampling it by a factor of two.

In the deblurring case, the low-pass filter corresponds to the modulation transfer function of the device, which we display in fig. 2. This filter has size $(2 m, 2 n)$ and its Fourier transform is small for frequencies larger than one half. We do not forget the prolate we used to generate the reference image and apply the same post-processing to all restored images (zoom + prolate convolution + subsampling).

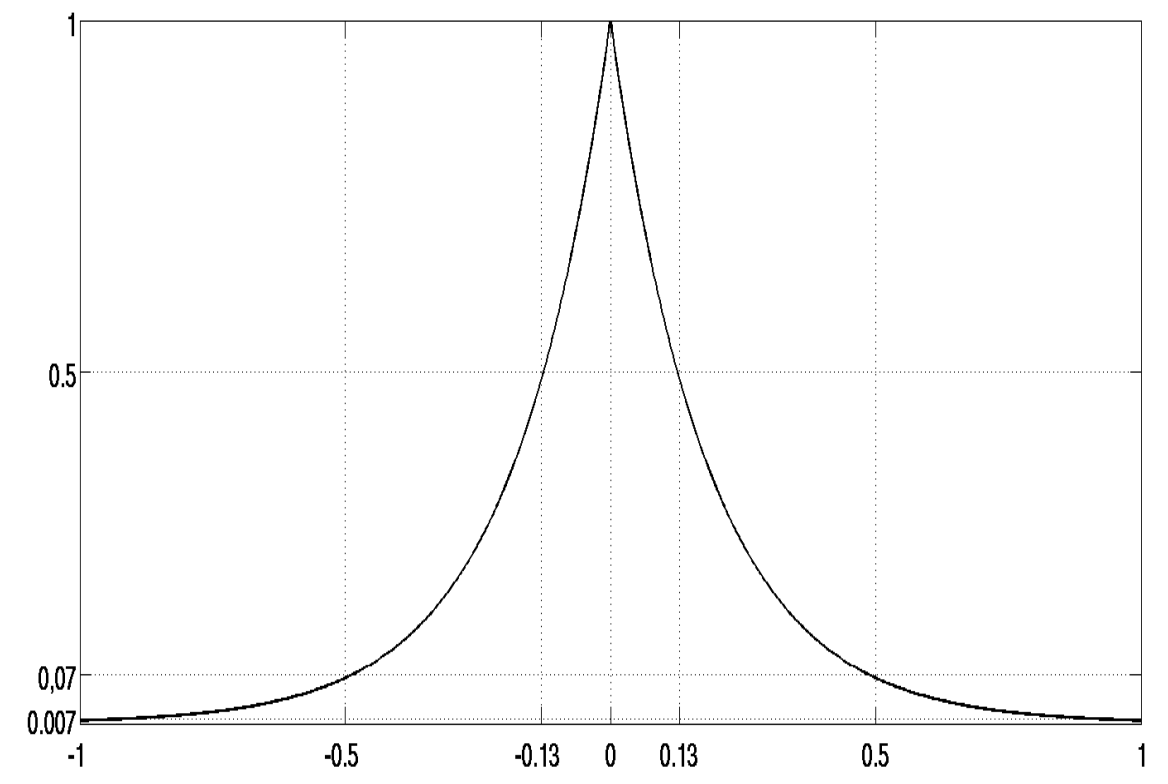

Fig. 2. MTF with normalized frequencies (size 2m.2n)

\subsubsection{Results}

We show some results with the following five methods

- ACT [1] : a truncated conjugate gradient with trigonometric polynomials, which stops when the constraint (4) is met.

- AC-S : the same as ACT in a spline space

- Tychonov-S : a quadratic regularizer with splines and global constraint as in [2], which we adapted to the deblurring problem. It is solved by a conjugate gradient and has automatic $\lambda$ update (as in the Uzawa loop of section 3.1.1).

- ACT+FAR [3] : a method with trigonometric polynomials, local constraints and a $\ell^{1}$ regularizer very close to TV.

- TV-S : our algorithm, which is a TV-regularized method with splines, global constraint and automatic $\lambda$ update (as in the Uzawa loop of section 3.1.1).

All these methods have a stopping criterion based on the noise variance $\sigma^{2}$. We considered different noise levels $\sigma \in$ $\{1,5,10\}$ (see tab. 1) and display images for $\sigma=5$ (see fig. 4). In the first column we displayed the restored image, in the second column the residue $(L . U-Z)$ (also known as method noise), and in the third column the Fourier transform of the residue. 


\begin{tabular}{||c||c|c|c||c||}
\hline & $\sigma=1$ & $\sigma=5$ & $\sigma=10$ & $\sigma=10$ \\
ACT & 39.16 & 31.07 & 28.42 & 28.68 \\
& $7.4 \mathrm{~s}$ & $5.5 \mathrm{~s}$ & $4.9 \mathrm{~s}$ & $4.8 \mathrm{~s}$ \\
\hline AC-S & 38.79 & 30.56 & 28.42 & 31.65 \\
& $0.9 \mathrm{~s}$ & 0.4 & $0.3 \mathrm{~s}$ & $0.1 \mathrm{~s}$ \\
\hline Tychonov-S & 38.65 & 30.89 & 28.61 & 31.97 \\
& $6.5 \mathrm{~s}$ & $2.3 \mathrm{~s}$ & $2.2 \mathrm{~s}$ & $1 \mathrm{~s}$ \\
\hline ACT+FAR & 40.51 & 32.08 & $\underline{28.90}$ & $\underline{32.85}$ \\
& $4 \mathrm{mn} 37 \mathrm{~s}$ & $2 \mathrm{mn} 35$ & $2 \mathrm{mn} 30 \mathrm{~s}$ & $1 \mathrm{mn} 12 \mathrm{~s}$ \\
\hline TV-S & $\frac{40.82}{3.9 \mathrm{~s}}$ & $\underline{32.26}$ & 28.85 & 32.28 \\
& $9.4 \mathrm{~s}$ & $3.2 \mathrm{~s}$ & $3.1 \mathrm{~s}$ \\
\hline
\end{tabular}

Table 1. PSNR and Computation times for a 259x259 image, blurry case with $\sigma \in\{1,5,10\}$ (first three columns) and unblurry case with $\sigma=10$ (last column)
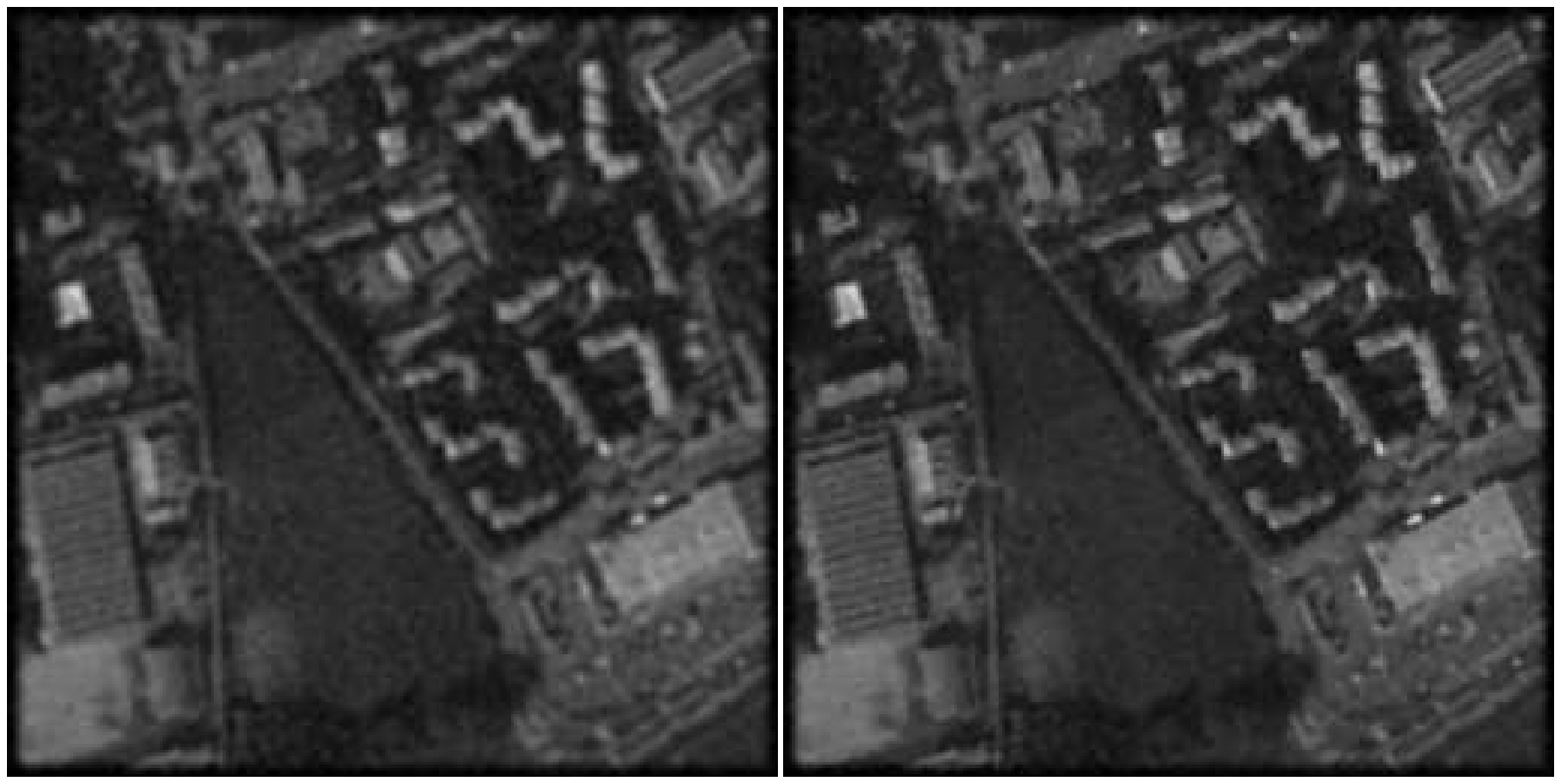

Fig. 3. Restored image with Tychonov-S method (left) and TV-S method (right) $(\sigma=5)$ 

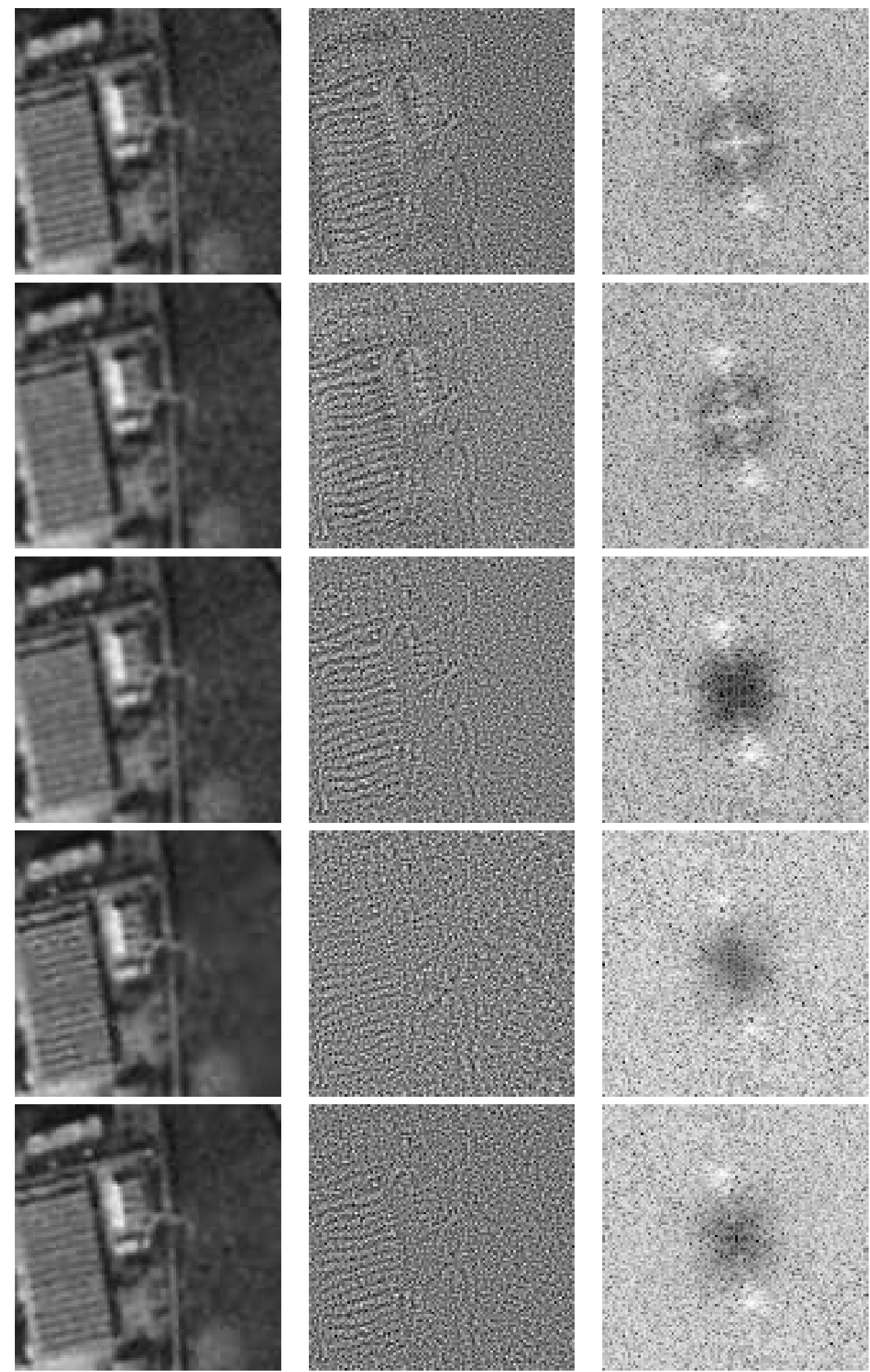

Fig. 4. Deblurring experiments $(\sigma=5)$, from top to bottom : ACT (1), AC-S (2), Tychonov-S (3), ACT+FAR (4), TV-S (5) (our method) 


\section{CONCLUSION}

These experiments show several interesting things. Our algorithm performs very well in deblurring, with equivalent or better results than ACT+FAR method, but much less computation time. It is a little bit slower than the quadratic regularization (Tychonov-S) but improves the global image quality, as it is less blurry and less noisy.

We also note that in the denoising case (last column of table 1) with an important noise level our algorithm is more efficient than a quadratic regularizer, although it is usually not the case for low noise levels. This can be explained by the erosion effect of total variation, which is too important with such a stopping condition, but could get fixed by adapting the target denoising level. (see [3])

Visual aspect of the result is also very good, we can see that the residue contains almost no structures (unlike ACT and AC-S). Smooth parts of the image are well restored, they look less noisy than Tychonov-S results (fig 3).

Deblurring has successfully been handled with this new spline formulation and computation time dramatically reduced compared to Fourier methods. We are enthusiastic towards more sophisticated methods including splines in irregular sampling, like non-local methods.

The authors would like to thank the CNES agency for kindly providing its copyrighted test image.

\section{REFERENCES}

[1] H. G. Feichtinger, K. Gröechenig, and T. Strohmer, "Efficient numerical methods in non-uniform sampling theory," Numerische Mathematik, vol. 69, no. 4, pp. 423-440, February 1995.

[2] M. Arigovindan, M. Sühling, P. Hunziker, and M. Unser, "Variational image reconstruction from arbitrarily spaced samples: A fast multiresolution spline solution," IEEE Transaction on Image Processing, vol. 14, no. 4, pp. 450-460, April 2005.

[3] G. Facciolo, A. Almansa, J. F. Aujol, and V. Caselles, "Irregular to regular sampling, denoising, and deconvolution," Multiscale Modeling \& Simulation, vol. 7, no. 4, pp. 1574-1608, 2009.

[4] A. Almansa, V. Caselles, G. Haro, and B. Rougé, "Restoration and zoom of irregularly sampled, blurred, and noisy images by accurate total variation minimization with local constraints," Multiscale Modeling \& Simulation, vol. 5, no. 1, pp. 235-272, 2006.

[5] P. L. Combettes and V. R. Wajs, "Signal recovery by proximal forward-backward splitting," Multiscale Modeling \& Simulation, vol. 4, no. 4, pp. 1168-1200, 2005.

[6] A. Chambolle, "An algorithm for total variation minimization and applications," Journal of Mathematical Imaging and Vision, vol. 20, no. 1-2, pp. 89-97, January 2004.

[7] J. J. Moreau, "Proximité et dualité dans un espace hilbertien," Bulletin de la S.M.F., vol. 93, pp. 273-299, 1965.

[8] P. L. Combettes, "Solving monotone inclusions via compositions of nonexpansive averaged operators," 2004.

[9] Y. Nesterov, "Gradient methods for minimizing composite objective function," , no. 2007076, 2007.

[10] P. Tseng and D. P. Bertsekas, "On the convergence of the exponential multiplier method for convex programming," Mathematical Programming, vol. 60, pp. 1-19, 1993. 